\title{
Letter to the Editor regarding Adams HL, Jaunoo SS. Hyperbilirubinaemia in appendicitis: the diagnostic value for prediction of appendicitis and appendiceal perforation. Eur J Trauma Emerg Surg. 2016; 42:249-52
}

\author{
P. Scholz ${ }^{1}$ U. Lenoir ${ }^{1} \cdot$ Y. Borbély ${ }^{1}$
}

Received: 10 June 2016 / Accepted: 16 June 2016 / Published online: 22 June 2016

(C) Springer-Verlag Berlin Heidelberg 2016

With great interest, we read the paper "Hyperbilirubinaemia in appendicitis: the diagnostic value for prediction of appendicitis and appendiceal perforation" [1]. Appendicitis with subsequent indication for appendectomy is the daily business for every general surgeon and additive measures to secure the diagnosis, especially, in patients with ambiguous clinical presentation are greatly appreciated. However, we do not see the advantage of an additional parameter with a sensitivity/specificity profile, such as bilirubin levels $(8 \% / 94 \%$ and $9.4 \% / 93 \%$ for acute and perforated appendicitis, respectively) measured in this paper. We wonder if those results changed the clinical decision and evaluation pathways in the authors' hospital, especially in light of already published papers covering this topic [2]. Two prospective trials, carried out and published prior to their paper, resulted in an even more devastating verdict over this parameter, concluding it to be of limited value in clinical routine and not to be recommended to predict perforation in appendicitis [3, 4].

Yet, this is not the main concern stipulating this letter. We simply fail to comprehend the arithmetic behind their results: the abstract section lists 484 patients $(86.9 \%)$ with histologically confirmed appendicitis, whereas the result section mentions 413 patients $(74.1 \%)$.

Furthermore, the method section terms laparotomy or conversion to laparotomy as exclusion criterion. In theory, the influence of a treatment method on a pre-operative diagnostic parameter should be negligible. In addition,
Table 1, specifying the characteristics of patients included in the study, reports only 154 patients to have undergone a laparoscopic approach. Following the criteria, the other 403 patients must have had an open procedure and should, therefore, be excluded.

We are very much looking forward to a clarification of these issues, as we were not able to figure out these inconsistencies ourselves.

\section{Compliance with ethical requirements}

Conflict of interest Philipp Scholz, Ursina Lenoir, and Yves Borbély declare that they have no conflict of interest.

\section{References}

1. Adams HL, Jaunoo SS. Hyperbilirubinaemia in appendicitis: the diagnostic value for prediction of appendicitis and appendiceal perforation. Eur J Trauma Emerg Surg. 2016;42:249-52.

2. Burcharth J, Pommergaard HC, Rosenberg J, Gogenur I. Hyperbilirubinemia as a predictor for appendiceal perforation: a systematic review. Scand J Surg. 2013;102:55-60.

3. Beltran MA, Mendez PE, Barrera RE, et al. Is hyperbilirubinaemia in appendicitis a better predictor of perforation than C-reactive protein?-a prospective study. Indian J Surg. 2009;71:265-72.

4. Muller S, Falch C, Axt S, et al. Diagnostic accuracy of hyperbilirubinaemia in anticipating appendicitis and its severity. Emerg Med J. 2015;32:698-702.
Y. Borbély

yves.borbely@insel.ch

1 Department of Visceral Surgery and Medicine, Inselspital, University Hospital of Bern, University of Bern,

Freiburgstrasse, 3010 Bern, Switzerland 